\title{
Construção e validação de uma escala de extroversão no modelo dos Cinco Grandes Fatores de Personalidade
}

\author{
Carlos Henrique Sancineto da Silva Nunes' \\ Cláudio Simon Hutr.
}

\begin{abstract}
Resumo
O presente estudo visou a construção e validação de construto de uma escala para a mensuração de extroversão no modelo dos Cinco Grandes Fatores de Personalidade. Nesse modelo, extroversão engloba traços que descrevem nível de comunicação, assertividade, gregariedade, busca por diversão, ente outros. A validade de construto da Escala Fatorial de Extroversão (EFE) contou com uma amostra de 1.084 pessoas de cinco estados brasileiros, de ambos os sexos, com nível de escolarização médio ou superior. Foram realizadas análises fatoriais para a verificação da dimensionalidade da EFE, sendo a solução de quatro fatores considerada a mais adequada. Os fatores extraídos foram denominados comunicação, altivez, assertividade e interações sociais, com alfas de Cronbach de 0,90; 0,78; 0,78 e 0,83, respectivamente. A escala geral apresentou uma consistência interna de 0,91.

Palavras-chave: Personalidade; Extroversão; Avaliação psicológica; Cinco Grandes Fatores.
\end{abstract}

\section{Development and validation of an extraversion scale in the Big Five Personality model}

\begin{abstract}
The present study was designed to develop and obtain construct validity for a scale to measure extraversion in the Big Five model. In this model, extraversion is comprised by traits that describe level of communication, assertivity, gregariousness, search for pleasure, among others. The participants in the construct validity study of the Extraversion Factorial Scale (EFE) were 1.084 individuals, from five states in Brazil, of both sexes, with high school or university level of education. Factor analyses were conducted to determine the EFE dimensions. A 4-factor solution was found to be more adequate. The factors found were named Communication, Pride, Assertiveness, and Social Interaction. Cronbach's alphas were $.90, .78, .78$, and .83 respectively. The general scale presented internal consistency level of .91 .
\end{abstract}

Keywords: Personality; Extraversion; Psychological assessment; Big Five Factors.

\section{Introdução}

Extroversão é um importante componente da personalidade humana que, no modelo dos Cinco Grandes Fatores (CGF), está relacionado às formas como as pessoas interagem com os demais e indica o quão comunicativas, falantes, ativas, assertivas, responsivas e gregárias elas são. Costa e Widiger (1993) indicaram que extroversão refere-se à quantidade e à intensidade das interações interpessoais preferidas, nível de atividade, necessidade de estimulação e capacidade de alegrar-se. Pessoas com elevados escores em extroversão (altas em extroversão) tendem a ser sociáveis, ativas, falantes, otimistas e afetuosas. Indivíduos com escores baixos em extroversão tendem a ser reservados (mas não necessariamente inamistosos), sóbrios, indiferentes, independentes e quietos. Introvertidos não são necessariamente pessoas infelizes ou pessimistas, mas não são dados aos estados de espírito exuberantes que caracterizam os extrovertidos.
Costa e McCrae (1980) têm argumentado que, em contextos clínicos, não somente extroversão, mas todos os cinco fatores podem ser úteis para a identificação de demandas de tratamento ou identificação de importantes sintomas de transtornos da personalidade. Mais especificamente, esses autores propuseram três motivos pelos quais tais medidas podem ser usadas nesses contextos: (1) avaliam estilos emocionais, interpessoais e motivacionais que podem ser de interesse aos clínicos; (2) oferecem um panorama compreensível do indivíduo que não pode ser obtido com a maioria dos instrumentos clinicamente orientados; (3) fornecem informações suplementares que podem ser úteis na seleção do tratamento e prognóstico dos casos.

Há também evidência na literatura (Widiger, Trull, Clarkin, Sanderson \& Costa, 2002) indicando que pacientes com diagnóstico de transtorno de personalidade histriônica, transtorno da personalidade esquizóide e esquizotípico, seguindo os critérios

\footnotetext{
${ }^{1}$ Endereço para correspondência:

Universidade Federal do Rio Grande do Sul - Instituto de Psicologia

Ramiro Barcelos, 2.600 - 90035-003 - Porto Alegre-RS

E-mail: carlos.sancineto@terra.com.br
} 
apresentados no DSM-IV (American Psychiatric Association, 1994), apresentam resultados muito diferenciados nas escalas de extroversão no modelo dos CGF. Eles destacam que indivíduos com diagnóstico de transtorno da personalidade histriônica, por exemplo, têm a tendência de expressar suas emoções de uma forma exagerada e teatral (com emoções excessivamente altas e positivas), são sexualmente provocativos e apresentam um comportamento de busca por atenção, consideram as relações mais íntimas do que realmente são e esforçam-se para ser o centro das atenções. Assim, de acordo com esses autores, o transtorno representa, em grande extensão, um caso extremo de extroversão. Extroversão envolve tendência de uma pessoa ser amigável e socialmente ousada, falante, afetuosa, festeira, ter muitos amigos, procurar ativamente contatos sociais, ser assertiva e dominante, enérgica, ativa, vigorosa, procurar estímulos fortes, envolver-se em riscos, ser otimista e divertida. Essas descrições de extroversão indicam o caso de uma pessoa prototipicamente histriônica.

Seguindo uma outra linha de pesquisa, Cooper, Agocha e Sheldon (2000) propuseram um modelo motivacional no qual foi testada a hipótese de que a personalidade influencia nos comportamentos de risco indiretamente de uma forma mediada, modelando a natureza e qualidade das experiências emocionais assim como os estilos característicos de coping relacionados a essas emoções. Esse modelo foi testado em uma amostra de 1.666 adultos jovens, com idades variando de 18 a 25 anos, e os resultados indicaram que os escores de neuroticismo e extroversão estão relacionados ao abuso de álcool e comportamentos sexuais de risco de formas distintas. Segundo os autores, indivíduos altos em neuroticismo apresentaram a tendência a engajar-se em comportamentos de risco como uma forma de lidar com estados de humor aversivos, enquanto indivíduos extrovertidos tendiam a envolver-se em comportamentos de risco como uma forma de realçar as experiências afetivas positivas. É importante salientar que as pesquisas nessa área têm apresentado resultados controversos e alguns autores (Loukas, Krull, Chassin \& Carle, 2000; Mulder, 2002), embora concordem com a existência de uma relação entre esses fatores e abuso e dependência de substâncias, apresentam modelos variados para a sua explicação.

No contexto do trabalho e das organizações, extroversão têm se mostrado uma importante variável, que se relaciona e prediz construtos úteis na área. Salgado (2002) realizou estudos metanalíticos que indicaram que os cinco fatores eram preditores de turnover. Todavia, ele não encontrou uma relação entre as dimensões dos CGF e absenteísmo ou acidentes. O autor indicou ainda que extroversão, realização e neuroticismo relacionam-se com a avaliação que supervisores fazem do desempenho no trabalho e o sucesso em programas de treinamento. É importante salientar que o desempenho no trabalho tem sido usualmente mensurado pela avaliação de supervisores e, em menor medida, pelo sucesso em treinamentos. Também é importante mencionar o trabalho de Bozionelos (2004), que investigou a relação entre os cinco fatores e comprometimento organizacional, verificando que extroversão e abertura explicam, em parte, o envolvimento de empregados com as empresas para as quais trabalham.

Waldman, Atwater e Davidson (2004) realizaram uma investigação sobre o papel do individualismo e os CGF na predição de desempenho em grupos de discussão sem líder. Os autores observaram que, entre os CGF, extroversão pode ser o fator mais relacionado com o desempenho em um contexto de grupo de discussão sem líder. Isso ocorreria por dois motivos básicos. Primeiro, em decorrência do desejo dos extrovertidos de obter interações sociais prazerosas, uma alta proporção deles pode resultar em uma perda de foco nas tarefas do grupo. Segundo, pelo fato de extrovertidos apresentarem uma tendência a ser dominantes e assertivos, pode emergir uma competição para o papel de liderança com prejuízo para a coesão do grupo e com aumento na incidência de conflitos Por outro lado, um grupo com poucas pessoas altas em extroversão pode não ser efetivo, pois pessoas dominantes e assertivas (pelo menos uma no grupo) são necessárias para preencher o papel de liderança. Os autores encontraram uma correlação positiva entre extroversão e desempenho de grupos sem liderança $(r=0,29, p<0,01)$.

A relação entre os CGF e desempenho no trabalho em pessoas de vários grupos ocupacionais e critérios de desempenho foi investigada por Barrick e Mount (1991). Os seus resultados indicaram que extroversão teve um melhor valor preditivo de desempenho para ocupações gerenciais e o de venda. No entanto, também indicaram que a relação entre personalidade e desempenho pode variar entre as ocupações e os critérios avaliados.

$\mathrm{Na}$ área do bem-estar subjetivo, Diener e Seligman (2002) compararam estudantes universitários que apresentavam níveis muito altos de felicidade (com pontos percentílicos iguais ou acima de 90 na escala de felicidade) com pessoas medianas e baixas nesse construto. As pessoas muito altas em felicidade eram muito sociáveis e românticas quando comparadas com as demais pessoas. Também eram mais altas em extroversão e socialização, mais baixas em neuroticismo 
e apresentaram níveis mais baixos no Minnesota Multiphasic Personality Inventory (MMPI). O grupo de pessoas mais felizes apresentou sentimentos positivos na maior parte do tempo, mas não de êxtase, e apresentou mau humor ocasional.

As múltiplas aplicações, tanto para a prática profissional como para inúmeras linhas de pesquisa enfatizam a importância do desenvolvimento de instrumentos válidos para a avaliação da personalidade no modelo dos Cinco Grandes Fatores no Brasil. Portanto, o presente estudo foi delineado para auxiliar a reduzir essa lacuna, desenvolvendo e validando um instrumento para a mensuração do fator extroversão.

\section{Método}

\section{Elaboração dos itens da Escala Fatorial de Extroversão}

O primeiro passo para a construção da Escala Fatorial de Extroversão no modelo dos CGF exigiu uma extensa pesquisa na literatura corrente sobre $O$ construto avaliado, com uma especial atenção a suas facetas (Goldberg, 1990; Hutz \& Nunes, 2001; O'Connor \& Dyce, 2002; Widiger, Trull, Clarkin, Sanderson \& Costa, 2002). Essas informações foram comparadas com os resultados de um estudo brasileiro para o desenvolvimento de marcadores de traços no modelo dos CGF (Hutz e cols., 1998) para a produção dos primeiros itens da escala. Não foi suficiente, contudo, a mera transformação dos adjetivos selecionados no referido estudo em assertivas, pois aqueles termos representavam traços de personalidade de uma forma muito ampla. Assim, um conjunto de itens do instrumento foi elaborado com base em descrições de comportamentos e atitudes relacionados a tais construtos.

Alguns itens foram elaborados tendo em vista estudos listados na literatura internacional (O'Connor \& Dyce, 2002; Trull \& McCrae, 2002; Widiger \& Frances, 2002; Widiger e cols., 2002) que têm verificado a relação entre a avaliação da personalidade pelos CGF e a descrição de transtornos de personalidade a partir de sistemas categóricos, como o DSM-IV (American Psychiatric Association, 1994). É possível verificar, nesses estudos, que alguns transtornos listados no DSM-IV são parcialmente contemplados pelos traços de personalidade agrupados nos fatores socialização, extroversão e neuroticismo. Foram escolhidos como referência alguns transtornos de personalidade que apresentavam uma relação mais estreita com extroversão, como transtorno de personalidade histriônica, transtorno de personalidade esquizóide e transtorno de personalidade de esquiva.
Uma ressalva deve ser feita, no entanto, em relação à amplitude dos traços cobertos pelos itens construídos tomando por base os sintomas dos quadros anteriormente enumerados. Claramente, esses quadros não podem ser compreendidos exclusivamente por uma única dimensão dos CGF. Muitos dos sintomas descritos como característicos do transtorno de personalidade histriônica, por exemplo, podem ser explicados por altos níveis de extroversão e neuroticismo e baixos níveis de socialização. Da mesma forma, muitos dos sintomas típicos do transtorno de personalidade obsessivo-compulsivo podem ser explicados por altos escores no fator realização e neuroticismo e baixos níveis em extroversão. Ainda assim, pode-se afirmar que alguns itens construídos a partir desse método representam adequadamente determinados fatores dentro do modelo dos CGF.

Apesar da grande variedade de traços que foram cobertos com os itens elaborados com base nos marcadores de traços compilados para o Brasil (Hutz \& cols., 1998) e de certos quadros contidos no DSM-IV (American Psychiatric Association, 1994), restaram ainda alguns aspectos descritos na literatura internacional a respeito de extroversão que não estavam contemplados até então. Assim, uma terceira fonte foi utilizada para a produção de itens. Trata-se de um projeto internacional de pesquisa em personalidade, denominado International Personality Item Pool (http://ipip.ori.org/ipip/index.htm).

Para a utilização dos itens disponibilizados na página do projeto IPIP, vários conjuntos de itens foram traduzidos para a língua portuguesa, buscando-se utilizar uma linguagem de fácil compreensão, na medida em que a população-alvo das escalas, adolescentes e adultos com ensino médio incompleto, não tem necessariamente que apresentar um alto nível cultural. A seguir, os itens foram submetidos à apreciação de quatro juízes familiarizados com o modelo dos CGF para verificar se, após a sua tradução, eles representavam adequadamente os fatores.

Após a elaboração da primeira versão, os itens para a Escala de Extroversão foram apresentados para onze pessoas, com diferentes níveis culturais e áreas de atuação, para avaliar a compreensão do vocabulário utilizado. Esse procedimento permitiu identificar alguns itens que eram confusos, incompreensíveis, ambíguos ou que apresentavam problemas variados na sua construção. Estes foram reelaborados ou eliminados. Após essa fase, a escala ficou com 140 itens. As instruções para aplicação das escalas também foram discutidas com juízes para garantir clareza e precisão. 
Adicionalmente, foi elaborado um roteiro de aplicação das escalas para garantir a padronização dos procedimentos para a coleta de dados. Esse roteiro foi encaminhado a todas as pessoas que se propuseram a participar dessa fase do processo e sofreu pequenos ajustes para garantir a sua compreensibilidade.

Um estudo piloto foi realizado com 62 estudantes ( $79 \%$ de mulheres e $21 \%$ de homens), com idade entre 17 e 45 anos $(\mathrm{M}=23,4$ e $\mathrm{DP}=5,9)$. Os resultados desse estudo levaram à eliminação de 30 itens, por diversas razões. Assim, a escala para os estudos de validade ficou com 110 itens.

\section{Validade de construto da EFE}

\section{Amostra}

A amostra para a avaliação das características psicométricas da EFE foi composta por 1.084 participantes de ambos os sexos (37,3\% homens e 62,7\% mulheres). O maior grupo da amostra foi composto por estudantes do curso de psicologia $(34,8 \%)$, seguido por estudantes secundaristas $(17,9 \%)$. Uma grande parcela do grupo avaliado indicou ser solteira $(83,1 \%)$ e com idade variando de 14 a 55 anos (média de 22,1 e desvio padrão de 6,42 anos). No entanto, é importante indicar a grande ocorrência de valores não informados nas variáveis contextuais. Isso ocorreu principalmente com as variáveis curso $(24,9 \%)$ e ocupação $(33,9 \%)$.

O número mínimo de participantes deste estudo foi calculado com o objetivo de gerar soluções fatoriais estáveis. Para tanto, foi utilizado o critério usualmente referido como "razão itens/sujeito". De acordo com este critério, para que se possa realizar uma análise fatorial confiável, é importante que a amostra seja de pelo menos cinco vezes o número de itens da escala a ser avaliada (Pasquali, 1999). Uma vez que a Escala Fatorial de Extroversão, na sua primeira versão, era composta por 110 itens, o número mínimo de participantes devia ser de 550 estudantes. Apesar dessa indicação, por não se conhecer o exato efeito das variáveis contextuais já citadas (sexo, idade, região, etc.), foi feita a opção de aumento desse número mínimo de participantes. Com isso, se de fato fosse verificado um efeito significativo de uma ou mais dessas variáveis, análises fatoriais independentes para cada grupo poderiam ser realizadas.

\section{Procedimentos de coleta de dados}

Os dados foram coletados em cinco estados brasileiros por pesquisadores e professores na área da Avaliação Psicológica que se propuseram a colaborar com essa fase do projeto (Tabela 1). A coleta foi coletiva, sendo usualmente realizada em salas de aula das instituições de ensino participantes (escolas de ensino médio, públicas e privadas; universidades públicas e privadas, cursos preparatórios para concursos, etc.). Nas instituições de ensino superior, foram escolhidas, preferencialmente, turmas de disciplinas que reuniam estudantes de vários cursos para aumentar a diversificação da amostra. Os estudantes, após serem informados dos objetivos do estudo, de que a participação era voluntária e da garantia de sigilo, receberam o caderno com os itens, a folha de respostas e instruções de preenchimento.

Foi solicitado consentimento esclarecido aos participantes deste estudo, obedecendo às regras de conduta ética na pesquisa com seres humanos. Aos estudantes que desejaram, foi oferecida uma devolução da avaliação realizada a partir de seus questionários.

Tabela 1 - Distribuição da amostra por estado

\begin{tabular}{ccc}
\hline Categoria & Freq. & $\%$ \\
\hline BA & 392 & 36,2 \\
RS & 358 & 33,0 \\
MG & 96 & 8,9 \\
SC & 71 & 6,5 \\
SP & 116 & 10,7 \\
Não informado & 51 & 4,7 \\
\hline Total & 1.084 & 100,0 \\
\hline
\end{tabular}

\section{Resultados}

Inicialmente, foi calculado o índice $\mathrm{KMO}$ para verificar a fatorabilidade dos itens da EFE. O resultado de 0,89 é considerado adequado para empregar técnicas de redução de dados e o teste de esfericidade de Bartlett foi significativo $(p<0,001)$, indicando a viabilidade da análise de componentes principais.

A primeira etapa da verificação das dimensões internas da EFE foi confeccionar um gráfico referido na literatura como scree plot, no qual os eigenvalues dos fatores são colocados no eixo Y de um gráfico cujo eixo X é o número dos fatores. A utilização desse gráfico para orientar os pesquisadores sobre o número de fatores a serem extraídos em um conjunto de dados foi proposto por Cattell (1966), de acordo com quem, o último fator a ser extraído pode ser visualizado em um scree plot como sendo aquele localizado no ponto de inflexão da curva. A Figura 1 foi elaborada segundo o procedimento sugerido.

Para a verificação da dimensionalidade da EFE, foram extraídas soluções fatoriais com 3, 4, 5 e 6 fatores, sendo que, pela regra de Cattell, a solução preferida deveria ser a de 3 dimensões. Como a maior parte dos itens criados supostamente representava o mesmo construto (o fator extroversão), considerou-se mais adequada a adoção da rotação direct oblimin, específica para a extração de fatores correlacionados. 
Uma questão que deve ser indicada é que, apesar de existirem inúmeras regras para a identificação do número de fatores a serem extraídas em uma análise fatorial, nenhuma delas é inquestionável ou pode ser considerada conclusiva sobre a questão. Em última análise, os fatores devem ser compreensíveis e teoricamente justificáveis.

\section{Scree Plot}

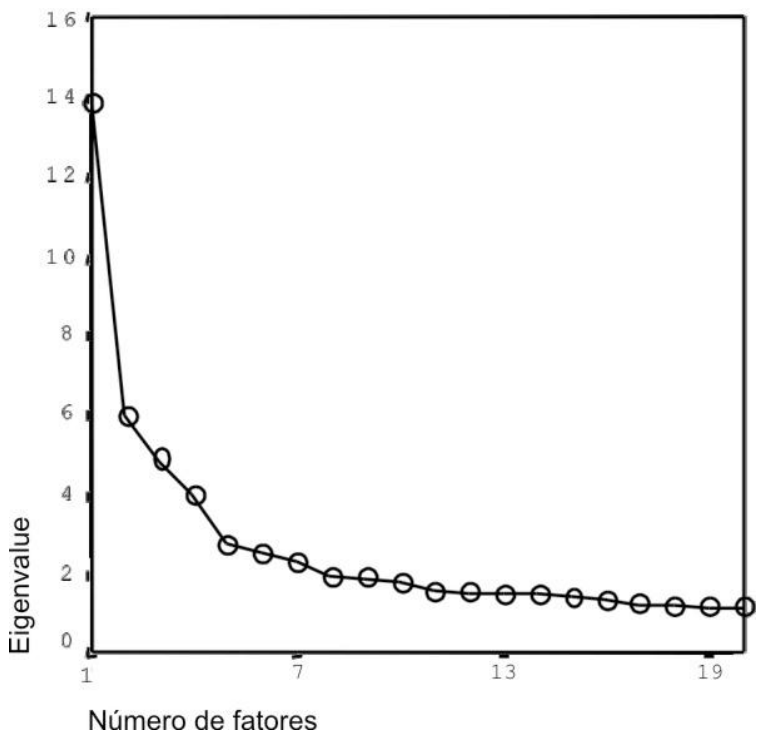

As soluções fatoriais extraídas foram verificadas levando-se em conta a pertinência dos fatores encontrados (quanto aos conteúdos) e características psicométricas das dimensões identificadas. De uma forma geral, a soluções de três e quatro fatores mostraram-se insatisfatórias por apresentarem, principalmente nos itens com menor carga fatorial, uma incompatibilidade de sentido destes com o conteúdo geral dos demais itens. As soluções com seis ou mais fatores resultaram em fatores que se diferenciavam por pequenas sutilezas, indicando que as soluções produzidas estavam excessivamente fragmentadas.

O melhor resultado foi conseguido quando foram extraídos cinco fatores que indicaram componentes denominados comunicação, altivez, assertividade, interações sociais e um quinto fator no qual se agruparam itens relacionados com abertura, que foram incluídos na versão preliminar da EFE. Esse fator, por ser constituído de itens fora do construto estudado, foi retirado da versão final da Escala Fatorial de Extroversão.

Como regra para inclusão dos itens nos fatores, foi determinado que teriam que apresentar no mínimo 0,35 de carga fatorial. Usualmente a linha de corte utilizada é de 0,30, mas, como o número de itens originais era muito elevado, essa opção permitiu selecionar os mais relacionados aos construtos avaliados. Não houve a ocorrência de itens com cargas fatoriais acima da linha de corte em mais de um fator.

$\mathrm{Na}$ Tabela 2 são apresentados os fatores extraídos, alguns exemplos de itens, seus conteúdos e a carga fatorial. Os fatores apresentaram, respectivamente, eigenvalues de 13,$88 ; 6,01,4,91$ e 4,04, que explicaram $12,62 \% ; 5,46 \%, 4,47 \%$ e $3,67 \%$ da variância total.

Tabela 2 - Exemplos de itens de comunicação, altivez, assertividade e interacõos sociais

\begin{tabular}{|c|c|c|}
\hline Item & Conteúdo & $\begin{array}{l}\text { Carga } \\
\text { fatorial }\end{array}$ \\
\hline & Comunicașão & \\
\hline 1 & $\begin{array}{l}\text { Costumo ficar calado quando estou } \\
\text { entre estranhos. }\end{array}$ & $-0,48$ \\
\hline 3 & $\begin{array}{l}\text { Costumo tomar a iniciativa e conversar } \\
\text { com os outros. }\end{array}$ & 0,45 \\
\hline 29 & Sou uma pessoa envergonhada. & $-0,62$ \\
\hline 100 & Tenho facilidade de me expressar. & 0,57 \\
\hline 110 & $\begin{array}{l}\text { Tenho vergonha de falar em grupo. } \\
\text { Assertividade }\end{array}$ & $-0,54$ \\
\hline 22 & Espero pela decisão dos outros. & $-0,43$ \\
\hline 26 & $\begin{array}{l}\text { Costumo agir com tranqüilidade diante } \\
\text { de situações novas. }\end{array}$ & 0,46 \\
\hline 39 & Tomo minhas decisões rapidamente. & 0,42 \\
\hline 106 & Desisto facilmente das coisas. & $-0,51$ \\
\hline 79 & $\begin{array}{l}\text { Deixo que os outros tomem as decisões } \\
\text { por mim. } \\
\text { Interacões sociais }\end{array}$ & $-0,46$ \\
\hline 14 & $\begin{array}{l}\text { Envolvo-me rapidamente com os } \\
\text { outros. }\end{array}$ & 0,37 \\
\hline 20 & Gosto de ter uma vida social agitada. & 0,57 \\
\hline 45 & Não consigo viver só. & 0,38 \\
\hline 50 & Transmito alegria aos outros. & 0,52 \\
\hline 59 & $\begin{array}{l}\text { Divirto-me quando estou entre muitas } \\
\text { pessoas. }\end{array}$ & 0,64 \\
\hline
\end{tabular}

Esse fator é composto por itens que descrevem o quão comunicativas e expansivas as pessoas acreditam que são. Pessoas com escores altos nessa escala usualmente apresentam facilidade para falar em público, tendem a falar mais sobre si mesmos e relatam ter facilidade de fazer novos conhecidos. Widiger e colaboradores (1993) e O'Connor e Dyce (2002) relatam que pessoas que apresentam transtomo da personalidade histriônica e dependente tendem a apresentar um nível de comunicação bem elevado, enquanto pessoas com transtorno da personalidade esquizóide e esquizotípico apresentam tendência oposta.

Esse fator é composto por itens que descrevem pessoas com uma percepção grandiosa sobre a sua 
capacidade e valor. Widiger e colaboradores (1993) e O'Connor e Dyce (2002) relatam que pessoas com transtorno da personalidade antissocial e narcisista tendem a identificar-se muito com esses itens, enquanto pessoas com transtorno da personalidade dependente apresentam tendência oposta.

Além disso, esses itens descrevem relatos comuns de pessoas com certos episódios de humor e transtorno da personalidade histriônica, e transtorno da personalidade de evitação (DSM-IV, American Psychiatric Association, 1994). Esses quadros apresentam configurações bem específicas, principalmente nos fatores extroversão e socialização (O'Connor \& Dyce, 2002; Widiger, Trull, Clarkin, Sanderson \& Costa, 2002) e, assim, pode-se questionar em que fator esses aspectos deveriam estar alocados dentro do modelo dos CGF.

No NEO-PI-R (Costa \& McCrae, 1992), existe um fator denominado modéstia dentro da Escala de Socialização (EFS), enquanto na construção das escalas de Extroversão (EFE) e EFS esses aspectos foram alocados na Escala de Extroversão. A baixa correlação entre altivez e os demais subfatores de extroversão indicam que os conteúdos avaliados pelo mesmo parecem diferenciar-se dos demais construtos. No entanto, quando é calculada a correlação do escore de altivez com a Escala Geral de Extroversão, essa é positiva e significativa $(r=0,55$; $p<0,001)$, enquanto a correlação entre o escore de altivez e a Escala Geral de Socialização foi negativa e mais baixa $(r=-$ $0,23 ; p<0,05)$. Esse resultado indica que a sua alocação neste fator parece ser pertinente.

Uma hipótese a ser explorada é a de que os conteúdos relacionados à altivez podem surgir por duas vias diferentes: (a) pessoas muito extrovertidas, além de iniciar e manter um nível de comunicação intenso com muitas pessoas, podem ter a percepção de que são especiais por conhecerem muitas pessoas, por circularem em muitos meios e se acharem muito íntimas dos outros; (b) pessoas com baixos níveis de socialização, além de apresentarem pouca empatia pelos demais, também tendem a descreverse como especiais, acima das regras e leis sociais, etc. Dessa forma, o que está sendo avaliado como altivez na EFE parece diferenciar-se da escala de Modéstia no NEO-PI-R (Costa \& McCrae, 1992), e na escala brasileira a relação dáse principalmente com Extroversão.

Esse fator é composto por itens que descrevem características como assertividade, liderança, nível de atividade e motivação. Essas características tendem a apresentar-se de em um nível muito baixo em pessoas com transtorno da personalidade dependente e de evitação, enquanto pessoas com transtorno obsessivo-compulsivo apresentam uma tendência oposta (O'Connor \& Dyce, 1993; Widiger, Trull, Clarkin, Sanderson \& Costa, 1993).
Esse fator descreve pessoas que buscam ativamente situações que permitam interações sociais como festas, atividades em grupo, etc. Pessoas com altos escores nessa escala tendem a ser gregárias e esforçam-se para manter contato com as pessoas conhecidas. O'Connor e Dyce (2002) e Widiger e colaboradores (2002) descreveram que pessoas com transtorno da personalidade histriônica apresentam esses comportamentos muito freqüentemente, enquanto pessoas com transtorno da personalidade anti-social tendem a identificar-se especificamente com os itens de busca por divertimento. Pessoas com transtorno da personalidade esquizotípica, esquizóide e de evitação tendem a apresentar um nível muito baixo de aderência a esses itens.

O próximo passo para a análise das características psicométricas da Escala Fatorial de Extroversão foi a verificação da consistência interna das suas subescalas. A Tabela 3 apresenta o alfa de Cronbach de nível de comunicação, altivez, assertividade e interações sociais bem como da EFE completo. É possível observar-se que a consistência interna das subescalas, apesar de bem abreviadas em relação ao seu número de itens, apresenta valores considerados adequados na literatura psicométrica (Pasquali, 1999, 2001).

Tabela 3 - Consistência interna das subescalas da EFE

\begin{tabular}{cccc}
\hline Fator & N. $^{\circ}$ itens & $\begin{array}{c}\text { Alfa de } \\
\text { Cronbach }\end{array}$ & $\begin{array}{c}\text { N. }^{\circ} \\
\text { participantes }\end{array}$ \\
\hline Comunicação & 19 & 0,90 & 1.032 \\
Altivez & 14 & 0,78 & 1.037 \\
Assertividade & 10 & 0,78 & 1.066 \\
Interações sociais & 14 & 0,83 & 1.041 \\
Escala geral & 57 & 0,91 & 958 \\
\hline
\end{tabular}

Com a verificação de que a Escala Fatorial de Extroversão e suas subescalas apresentam características psicométricas adequadas para a avaliação de seus construtos, foi necessário verificar quais variáveis apresentam influência sobre as mesmas. As pesquisas com a validação da Escala Fatorial de Neuroticismo (Hutz \& Nunes, 2001; Nunes, 2000) indicaram que o sexo do respondente apresentou uma forte influência sobre os resultados observados tanto na escala geral quanto nos seus subfatores, o que justificou a elaboração de tabelas específicas para homens e mulheres. As variáveis estado, estado civil, escolaridade e idade não apresentaram uma influência estatisticamente significativa sobre a escala. Assim, para verificar-se a influência do sexo e estado do respondente, foi 
realizada uma análise GLM para medidas repetidas. Para tanto, um fator geral de extroversão foi gerado pela definição de quatro níveis (E1, E2, E3 e E4) e os fatores entre os sujeitos foram as variáveis sexo e estado. Essa análise indicou que a variável sexo do respondente gerou perfis diferenciados para extroversão enquanto a variável estado não apresentou diferença significativa para o resultado de extroversão. As duas variáveis, quando combinadas, não geram diferenças estatisticamente significativas sobre extroversão. A diferença encontrada entre sexos é muito pequena e a significância estatística decorre do tamanho da amostra, não tendo implicações clínicas. Isso fica claro ao se observar o tamanho do efeito para a escala geral $(d=0,11)$ e a Tabela 4 , onde são apresentadas as médias e medianas por sexo para cada fator.

Tabela 4 - Médias e mediana e desvio padrão das subescalas e escala geral da EFE por sexo

\begin{tabular}{rrrrrrrrrrr}
\hline & \multicolumn{4}{c}{ Homens $(\mathrm{n}=399)$} & \multicolumn{4}{c}{ Mulheres (n=699) } \\
\hline & E1 & E2 & E3 & E4 & EFE & E1 & E2 & E3 & E4 & EFE \\
Média & 4,4 & 3,8 & 5,1 & 5,0 & 18,2 & 4,4 & 3,5 & 4,9 & 5,1 & 17,9 \\
Mediana & 4,4 & 3,8 & 5,1 & 5,1 & 18,3 & 4,4 & 3,5 & 4,9 & 5,2 & 18,1 \\
Desvio padrão & 1,11 & 0,94 & 0,92 & 0,92 & 2,70 & 1,14 & 0,92 & 0,98 & 0,95 & 2,77 \\
\hline
\end{tabular}

E1= Comunicação

$\mathrm{E} 2=$ Altivez

E3=Assertividade

E4=Interações sociais

As correlações entre idade do respondente e os escores observados nas escalas da EFE são apresentadas na Tabela 5. Como se observa, as correlações entre idade e E1 e E3 são significativas. Porém, o valor dessas associações é muito baixo e também decorre do tamanho da amostra. Por isso não parece haver necessidade de elaborar tabelas normativas para diferentes faixas etárias.

Tabela 5 - Correlação entre a idade e as escalas da $\mathrm{EFE}-\mathrm{N}=1,057$

\begin{tabular}{rccccc}
\hline \multicolumn{1}{c}{ E1 } & E2 & E3 & E4 & EFE \\
\hline Idade & $0,11^{*}$ & $-0,03$ & 0,05 & $-0,11^{*}$ & 0,01 \\
\hline$*^{2} 0,01$ & & & & &
\end{tabular}

\section{Discussão}

As análises realizadas no presente estudo indicam que a escala construída apresenta boas características psicométricas, com subdimensões compatíveis com as listadas na literatura internacional (Widiger \& Trull, 1992). Os subfatores apresentaram uma elevada consistência interna e associaram-se entre si de acordo com os resultados geralmente obtidos nas pesquisas na área.

É importante relembrar que, apesar de sua amplitude e representação regional, os dados do presente estudo foram coletados com base em uma amostra não-aleatória. A rigor, a amostra não pode ser considerada representativa da população brasileira. O estudo realizado deve ser ampliado para amostras em todas as regiões do país e em diversas classes sociais e culturais para produzir normas nacionais. Ainda assim, a replicação independente dos achados apresentados é fundamental para aumentar a confiança no instrumento.

Além disso, também são necessários estudos para identificar os contextos nos quais a EFE pode ser utilizada adequadamente, especialmente para fins de diagnóstico ou na área organizacional. Espera-se que com a divulgação deste instrumento se torne possível pesquisadores da área desenvolverem pesquisas com grupos variados, especialmente grupos clínicos, com o objetivo de determinar a validade de critério da escala e começar a estabelecer pontos de corte com informação sobre falsos positivos e negativos.

\section{Referências}

American Psychiatric Association (1994). Diagnostic and statistical manual of mental disorders: DSM-IV (4th ed.). Washington, DC: American Psychiatric Association.

Barrick, M. R. \& Mount, M. K. (1991). The big five personality dimensions and job performance: A metaanalysis. Personnel Psychology, 44, 1-26.

Bozionelos, N. (2004). The big five of personality and work involvement. Journal of Managerial Psychology, 19, 69-81.

Cattell, R. B. (1966). The meaning and strategic use of factor analysis. Em R. B. Cattell (Org.). Handbook of multivariate experimental psychology (pp. 174-243). Chicago: Rand McNally. 
Cooper, M. L., Agocha, V. B. \& Sheldon, M. S. (2000). A motivational perspective on risky behaviors: The role of personality and affect regulatory processes. Journal of Personality, 6, 1059-1088.

Costa, P. T., Jr. \& McCrae, R. R. (1980). Influence of extraversion and neuroticism on subjective well-being. Journal of Personality and Social Psychology, 38, 668-678.

Costa, P. T., Jr. \& McCrae, R. R. (1992). Revised NEO Personality Inventory (NEO-PI-R) and Five Factor Inventory (NEO-FFI) professional manual. Odessa, FL: Psychological Assessment Resources.

Costa, P. T., Jr. \& Widiger, T. A. (1993). Introduction. Em P. T. Costa \& T. A. Widiger (Orgs.). Personality disorders and the Five-Factor Model of Personality (pp. 1-10). Whashington, DC: American Psychological Association.

Diener, E. \& Seligman, M. E. P. (2002). Very happy people. Psychological Science, 13, 80-84.

Goldberg, L. R. (1990). An alternative "description of personality": The Big-Five Factor Structure. Journal of Personality and Social Psychology, 59, 1216-1229.

Hutz, C. S. \& Nunes, C. H. S. S. (2001). Escala Fatorial de Neuroticismo. São Paulo: Casa do Psicólogo.

Hutz, C. S., Nunes, C. H., Silveira, A. D. Serra, J. \& Anton, M. (1998). O desenvolvimento de marcadores para a avaliação da personalidade no modelo dos cinco grandes fatores. Psicologia: Reflexão e Crítica, 11, 395-409.

Loukas, A., Krull, J. L., Chassin, L. \& Carle, A. C. (2000). The relation of personality to alcohol abuse / dependence in a high-risk sample. Journal of Personality, $68,1153-1175$.

Mulder, R. T. (2002). Alcoholism and personality. Australian and New Zealand Journal of Psychiatry, 36, 44-52.

Nunes, C. H. S. S. (2000). A construção de um instrumento de medida para o fator neuroticismo / estabilidade emocional dentro do modelo de personalidade dos cinco grandes fatores. (Dissertação de Mestrado). Porto Alegre, RS: UFRGS Instituto de Psicologia.

O’Connor, B. P. \& Dyce, J. A. (1993). Test of General and Specific Models of Personality Disorder Configuration. Em P. T. Costa \& T. A. Widiger (Orgs.). Personality disorders and the Five-Factor Model of Personality (pp. 223-246). Washington, DC: American Psychological Association.

O’Connor, B. P. \& Dyce, J. A. (2002). Test of General and Specific Models of Personality Disorder Configuration. Em P. T. Costa \& T. A. Widiger (Orgs.). Personality disorders and the Five-Factor Model of Personality ( $2^{\mathrm{a}}$ ed., pp. 223-248). Washington, DC: American Psychological Association.
Pasquali, L. (1999). Testes referentes a construto: teoria e modelo de construção. Em L. Pasquali (Org.). Instrumentos psicológicos: manual prático de elaboração (pp. 3771). Brasília, DF: Laboratório de Pesquisa em Avaliação e Medida - LabPAM.

Pasquali, L. (2001). Técnicas de Exame Psicológico - TEP: manual. São Paulo, SP: Casa do Psicólogo; Conselho Federal de Psicologia.

Salgado, J. F. (2002). The big five personality dimensions and counterproductive behaviors. International Journal of Selection and Assessment, 10(1/2), 117-125.

Trull, J. T. \& McCrae, R. R. (2002). A five-factor perspective on personality disorder research. Em P. T. Costa \& T. A. Widiger (Orgs.). Personality disorders and the five-factor model of personality ( $2^{\mathrm{a}}$ ed., pp. 45-58). Whashington, DC: American Psychological Association.

Waldman, D. A., Atwater, L. E. \& Davidson, R. A. (2004). The role of individualism and the Five-Factor Model in the prediction of performance in a leaderless group discussion. Journal of Personality, 72, 1-28.

Widiger, T. A. \& Trull, T. J. (1992). Personality and psychopatology: An application of the Five-Factor Model. Journal of Personality, 60, 363-393.

Widiger, T. A. \& Frances, A. J. (2002). Toward a dimensional model for the personality disorders. Em P. T. Costa \& T. A. Widiger (Orgs.). Personality disorders and the Five-Factor Model of Personality (2 ${ }^{\mathrm{a}}$ ed., pp. 23-44). Washington, DC: American Psychological Association.

Widiger, T. A., Trull, T. J., Clarkin, J. F., Sanderson, C. \& Costa, P. T. (1993). A description of the DSM-III-R and DSM-IV personality disorders with the FiveFactor Model of Personality. Em P. T. Costa \& T. A. Widiger (Orgs.). Personality disorders and the Five-Factor Model of Personality (pp. 41-56). Washington, DC: American Psychological Association.

Widiger, T. A., Trull, T. J., Clarkin, J. F., Sanderson, C. \& Costa, P. T. (2002). A description of the DSM-IV personality disorders with the five-factor model of personality. Em P. T. Costa \& T. A. Widiger (Orgs.). Personality disorders and the Five-Factor Model of Personality ( $2^{\mathrm{a}}$ ed., pp. 89-102). Washington, DC: American Psychological Association.

Recebido em agosto de 2005 Reformulado em agosto de 2006 Aprovado em outubro de 2006 
Sobre os autores:

Carlos Henrique Sancineto da Silva Nunes é psicólogo, doutor em Psicologia pelo Departamento de Psicologia do Desenvolvimento da Universidade Federal do Rio Grande do Sul, docente na Faculdade Ruy Barbosa e secretário do Instituto Brasileiro de Avaliação Psicológica (IBAP).

Cláudio Simon Hutz é psicólogo, professor titular do Instituto de Psicologia da Universidade Federal do Rio Grande do Sul, PhD pela University of Iowa (USA), pós-doutorado na Arizona State University (USA), coordenador do Laboratório de Mensuração da UFRGS. 
